\title{
A viral protein kinase drug target for tumors?
}

\author{
Richard F. Ambinder \\ Department of Oncology, Johns Hopkins School of Medicine, Baltimore, Maryland, USA.
}

\begin{abstract}
The human gammaherpesviruses, Epstein-Barr virus (EBV) and Kaposi's sarcoma-associated herpesvirus (KSHV), are both associated with tumors. Standard antiviral therapies are ineffective at treating these tumors. A serine/threonine kinase important for viral replication is conserved across the herpesviruses. Expression of the KSHV protein kinase in transgenic mice under the control of a ubiquitin promoter was associated with B cell lymphoproliferative disease and lymphoma. If the viral protein kinase is important in the pathogenesis of KSHV lymphoproliferative disease or lymphoma, the kinase may present a very good target for pharmacologic therapies.
\end{abstract}

\section{Human gammaherpesviruses and tumors}

There are two human gammaherpesviruses, and both are associated with malignancies: EBV and Kaposi's sarcomaassociated herpesvirus (KSHV), also known as human herpesvirus 8 (HHV8). Indeed, both were originally discovered in tumors. EBV was identified in endemic (African) Burkitt lymphoma $(1,2)$. Subsequent studies showed the virus to be consistently associated with undifferentiated nasopharyngeal carcinoma, nasal $\mathrm{NK} / \mathrm{T}$ cell lymphoma, early posttransplant lymphoma, and AIDS primary central nervous system lymphoma and variably associated with Hodgkin lymphoma, diffuse large B cell lymphoma, peripheral $\mathrm{T}$ cell lymphoma, and gastric lymphoma. KSHV was first identified in Kaposi's sarcoma and subsequently identified in primary effusion lymphoma, a highly aggressive and difficult to treat B cell lymphoma that typically presents as a malignant effusion and is almost exclusively seen in patients with HIV (3). In addition, KSHV is consistently associated with Castleman's disease in HIV-infected patients. Although not considered neoplastic because it is polyclonal, Castleman's disease is a B cell lymphoproliferative disease. Both viruses are trans- mitted in saliva, and both establish latency in B lymphocytes.

Although there are antivirals that inhibit KSHV and EBV virion production, these have proven ineffective for the treatment of tumors. Available pharmacologic agents inhibit the viral DNA polymerases required for the production of new virions in lytic infection, but do not inhibit replication of viral genomes in latently infected tumor cells. Replication of viral genomes in latently infected tumor cells is carried out by the host cell polymerase. Similarly, tumor virus genomes integrated into the host cell genome are also replicated by host cell DNA polymerase. However, in contrast with the human papillomaviruses and Merkel cell polyomavirus, the gammaherpesvirus genomes are generally not found to be integrated in tumor DNA. These large double-stranded DNA genomes are present in cells, including most tumor cells, as nuclear plasmids or episomes. Their persistence in dividing cells requires viral proteins that allow them to divide in synchrony with the cell cycle and to be partitioned among daughter cells. Approaches to specifically inhibit the function of these viral proteins are still in their infancy, although there are some promising developments (4).

Related Article: p. 2519

Conflict of interest: The author has declared that no conflict of interest exists.

Reference information: / Clin Invest. 2018;128(6):2197-2198. https://doi.org/10.1172/JCI121080.

\section{Viral serine/threonine kinases}

In addition to the gammaherpesviruses that are associated with tumors, there are $\alpha$ (herpes simplex and varicella-zoster virus) and $\beta$ herpesviruses (cytomegalovirus, HHV6, HHV7). Viral infections are associated with all manner of human ills, from fever blisters to intellectual disability, pneumonitis, and birth defects (5). All mammalian herpesviruses reprogram host cell gene expression, sense the state of the cell cycle, alter that state, and activate lytic gene expression to produce new virions $(5,6)$. The herpesviruses encode latency genes that are unique to each subfamily, while many of the lytic genes, such as those encoding proteins involved in lytic viral genome replication or that are structural components of the virion, are conserved. Among the conserved replication proteins is a serine/threonine protein kinase with structural similarity to the cellular kinase cyclin-dependent kinase 2 (cdk2) (7). These viral protein kinases phosphorylate CDK cellular targets as well as viral protein targets. They are more promiscuous than cellular CDKs and phosphorylate some other targets as well.

The human cytomegalovirus (HCMV) protein kinase UL97 phosphorylates ganciclovir, beginning a phosphorylation series that leads to ganciclovir triphosphate, which inhibits viral and cellular DNA polymerases. This is the basis for the clinical efficacy of ganciclovir in the treatment of HCMV. Ganciclovir also has some activity in EBV and KSHV infection. An inhibitor of UL97, maribavir is in clinical trials for the treatment of HCMV. Protein kinase activity appears to be required for egress of virus from the nucleus.

In this issue of the JCI, investigators created two heterozygous transgenic mouse lines expressing the KSHV protein kinase under the control of a ubiquitin promoter (8). The authors document broad protein kinase expression in a variety of tissues, but focus on the B cell compartment. The mice showed increased numbers of germinal center B cells even in the absence of antigen stimulation and 
a substantially increased incidence of $\mathrm{B}$ cell lymphoma or lymphoproliferative disease in comparison with nontransgenic controls. The resultant tumors expressed germinal center and post-germinal center markers. Tumor cells were highly proliferative, produced inflammatory cytokines, and expressed the viral protein kinase. Clonality studies indicated that some of the tumors were oligoclonal, while others were polyclonal. Thus, the KSHV protein kinase can clearly drive cell proliferation and contribute to a phenotype that resembles primary effusion lymphoma. It should be noted that as of yet, there is no direct evidence that the protein kinase is what is driving lymphoproliferation in patients. In this regard, it is worth noting that there is evidence that the KSHV protein kinase is under the regulation of a promoter that includes hypoxia-responsive elements and thus this protein may be expressed under hypoxic conditions, such as those associated with primary effusion lymphoma even when other viral lytic proteins are not expressed $(9,10)$.

\section{Conclusions}

The findings warrant attention because we have gotten very good at inhibiting various cellular and viral kinases, including CDKs. Several CDK inhibitors have been licensed to treat cancer and many more are in trial (11). In addition, as noted above, there is already an antiviral that inhibits the HCMV protein kinase in clinical trials (12). With large libraries of kinase inhibitors available, it seems likely that effective inhibitors of the KSHV protein kinase have already been synthesized and that if the viral protein kinase is indeed an important contributor to the development or maintenance of KSHV lymphoproliferative disease, drug therapies that will prevent or treat KSHV-driven lymphoproliferative disease will emerge.

\section{Acknowledgments}

Thisworkwas supported byUM1CA121947, P30AI094189, and P30CA006973.

Address correspondence to: Richard F. Ambinder, Department of Oncology, Johns Hopkins School of Medicine, Cancer Research Building, Room 389, 1650 Orleans Street, Baltimore, Maryland 21287, USA. Phone: 410.955.8839; Email: rambind1@jhmi.edu.

1. Kanakry JA, Ambinder RF. Human herpesviruses: malignant lymphoma. In: Kaslow RA, Stanberry LR, Le Duc JW, eds. Viral Infections of Humans Epidemiology and Control. 5th ed. New York, New York, USA: Springer; 2014:933-951.

2. Young LS, Yap LF, Murray PG. Epstein-Barr virus: more than 50 years old and still providing surprises. Nat Rev Cancer. 2016;16(12):789-802.

3. Cesarman E. Gammaherpesviruses and lymphoproliferative disorders. Annu Rev Pathol. 2014;9:349-372.

4. Gianti E, Messick TE, Lieberman PM, Zauhar RJ. Computational analysis of EBNA1 “druggability" suggests novel insights for Epstein-Barr virus inhibitor design. J Comput Aided Mol Des. 2016;30(4):285-303.

5. Arvin A, et al. Human Herpesviruses: Biology, Therapy, and Immunoprophylaxis. Cambridge, United Kingdom: Cambridge University Press; 2007.

6. Li R, et al. Conserved herpesvirus kinases target the DNA damage response pathway and TIP60 histone acetyltransferase to promote virus replication. Cell Host Microbe. 2011;10(4):390-400.

7. Roller RJ, Baines JD. Herpesvirus nuclear egress. In: Osterrieder K, ed. Cell Biology of Herpes Viruses. New York, New York, USA: Springer; 2017:143-169.

8. Anders PM, Montgomery ND, Montgomery SA, Bhatt AP, Dittmer DP, Damania B. Human herpesvirus-encoded kinase induces B cell lymphomas in vivo. J Clin Invest. 2018;128(6):2519-2534.

9. Haque M, Davis DA, Wang V, Widmer I, Yarchoan R. Kaposi's sarcoma-associated herpesvirus (human herpesvirus 8) contains hypoxia response elements: relevance to lytic induction by hypoxia. J Virol. 2003;77(12):6761-6768.

10. Davis DA, Singer KE, Reynolds IP, Haque M, Yarchoan R. Hypoxia enhances the phosphorylation and cytotoxicity of ganciclovir and zidovudine in Kaposi's sarcoma-associated herpesvirus infected cells. Cancer Res. 2007;67(14):7003-7010.

11. Sherr CJ, Bartek J. Cell cycle-targeted cancer therapies. Annu Rev Cancer Biol. 2017;1:41-47.

12. Sharma M, et al. Human cytomegalovirus UL97 phosphorylates the viral nuclear egress complex. J Virol. 2015;89(1):523-534. 